\title{
A Virtual Handwriting Tablet Based on Pen Shadow Cues
}

\author{
Chin-Shyurng Fahn, Bo-Yuan Su, and Meng-Luen Wu \\ Department of Computer Science and Information Engineering \\ National Taiwan University of Science and Technology \\ Taipei, Taiwan 10607, Republic of China \\ \{csfahn, a9815001,D10015015\}@mail.ntust.edu.tw
}

\begin{abstract}
The handwriting tablet is an electronic product, which is a kind of human-computer interfaces acting as a computer input device comprising a set of a special pen and a tablet. The user holds the pen to draw contents within a region of the tablet as inputs, which imitates handwriting and is a replacement of mouse inputs. Some handwriting tablets not only imitate the handwriting and mouse functions, but also detect the pen tilts and pressures. The tilt and pressure information can be applied to some drawing software which can also render the thickness and depth of strokes. However, since the handwriting tablet is a piece of precise equipment, it has some drawbacks- fragile, not easy to carry, and the weight is often heavy. Therefore, in this paper, we propose a new concept based on the computer vision technology to simulate the handwriting tablet. We put a rectangular plane in the FOV of a video camera to emulate a tablet, and use a conventional pen to emulate the stylus. Many experiments have been made for evaluating the effectiveness of the proposed methods. The performance of such a virtual handwriting tablet is very satisfactory and encouraged.
\end{abstract}

Keywords: virtual handwriting tablet, shadow cues, computer vision, humancomputer interface.

\section{$1 \quad$ Introduction}

With the evolution of information technology, there are various means of humancomputer interfaces. In order to make computers more convenient and interactive to control, several ways of controls have been developed, such as joysticks, hand gestures, and pens. Nowadays, human-computer interfaces without keyboards and mice are a trend because they provide users different experiences. For example, in racing games, wheel shaped joysticks are created to simulate handlers for actual cars, which gives players a more entertaining experience. Input texts via handwriting tablets supplies users a feel of writing.

The handwriting tablet is a kind of computer input devices, which is an electronic product composed of a special pen and a tablet. The user holds the pen to draw contents within a region of the tablet as inputs, which imitates handwriting and replaces mouse inputs. Some handwriting tablets not only imitate the handwriting and 
mouse functions, but also detect pen tilts and pressures. Both the tilt and pressure information can be applied to some drawing software that the thickness and the depth of strokes can be also rendered [1].

However, since the handwriting tablet is a piece of precise equipment, it has some drawbacks- fragile, not easy to carry, and too heavy. Therefore, we adopt the computer vision technology together with a few materials to simulate the handwriting tablet. This novel concept is to develop a virtual handwriting tablet as shown in Figure 1, where we put a rectangular plane (that can be a cardboard, corrugated paper, and so on) in the FOV of a video camera to emulate a tablet, and use a conventional pen to emulate the stylus (the pen for handwriting tablet) [2],[3].

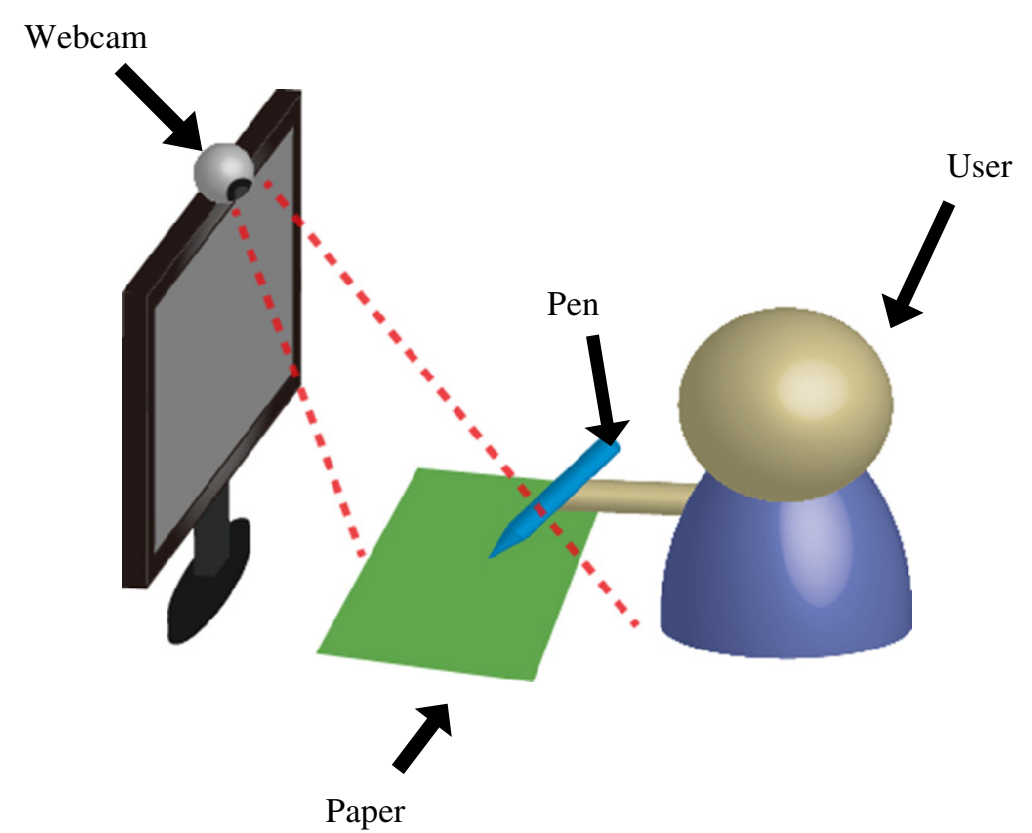

Fig. 1. The concept of the virtual handwriting tablet.

The development of such a virtual handwriting tablet is divided into three parts, including tablet detection, stylus detection, and pen shadow detection. First, the user selects an object in the FOV as the tablet, and the system determines whether the selected object meets the conditions on the form of a tablet. After the tablet object is confirmed, the system detects a pen in the tablet region. If so, the shadow of the pen is then detected. The tablet, pen, as well as shadow information are saved for the system. Figure 2 graphically shows the main flowchart of our virtual handwriting tablet system. 


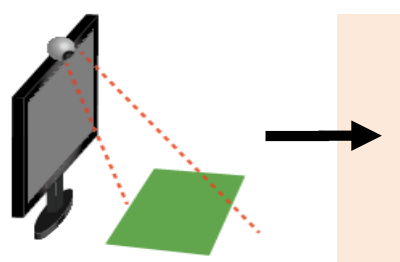

The FOV of a camera

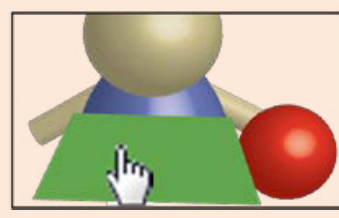

Select an object as a tablet

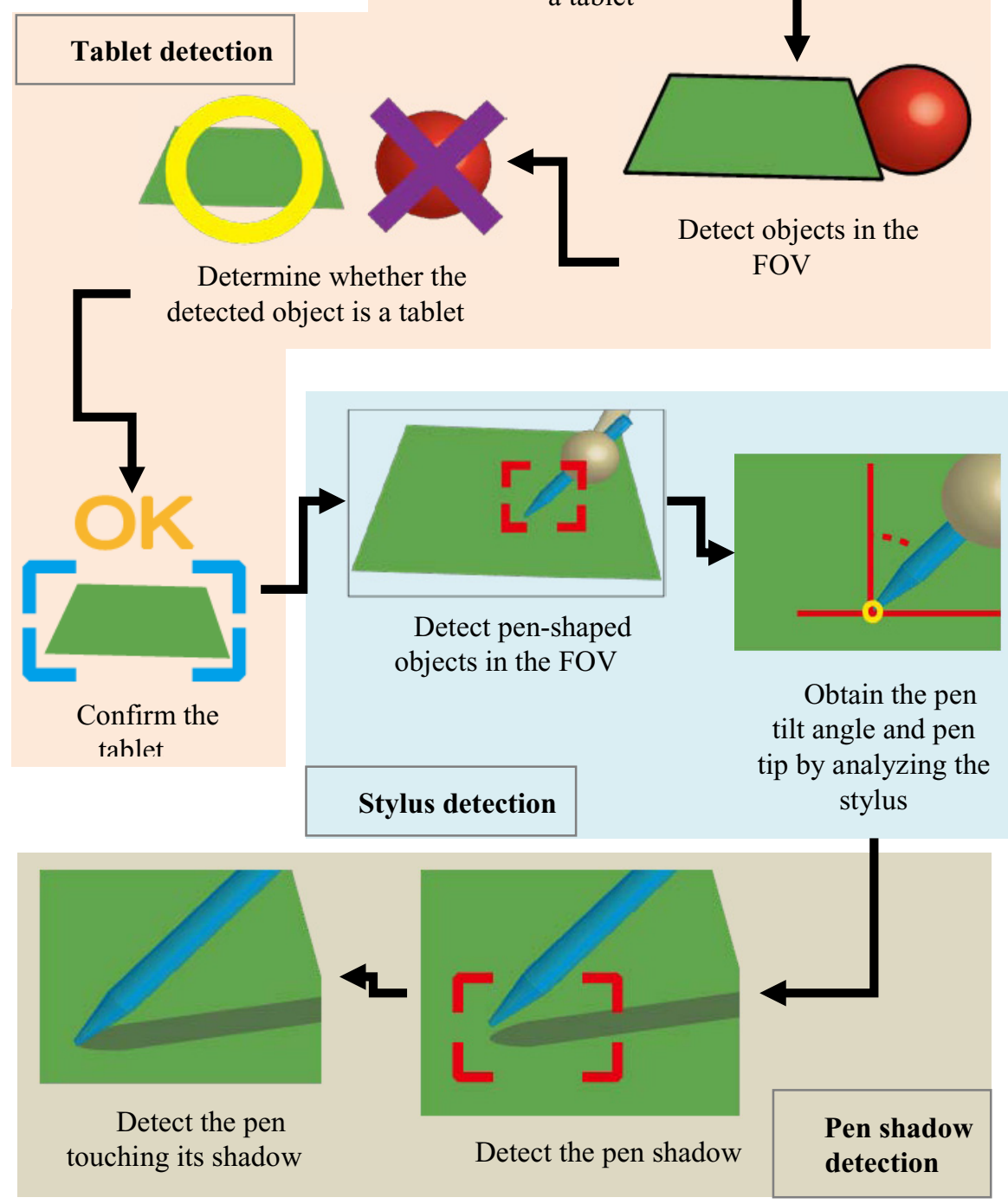

Fig. 2. The main flowchart of our virtual handwriting tablet system 


\section{The Relation of Pen and Its Shadow}

The following elaborates how we acquire the distance between the pen and its shadow used for the detection of a pen touching or detaching a tablet. After the area of the shadow is obtained, we can use the bounding box of the shadow to get the boundary and position of the area. By analyzing the variation of the distance from a pen to its shadow, we can detect the relation between the pen and the tablet. Figure 3 illustrates the pen under different tilt degrees in several sampling frames that the pen approaches the tablet and leaves it gradually.

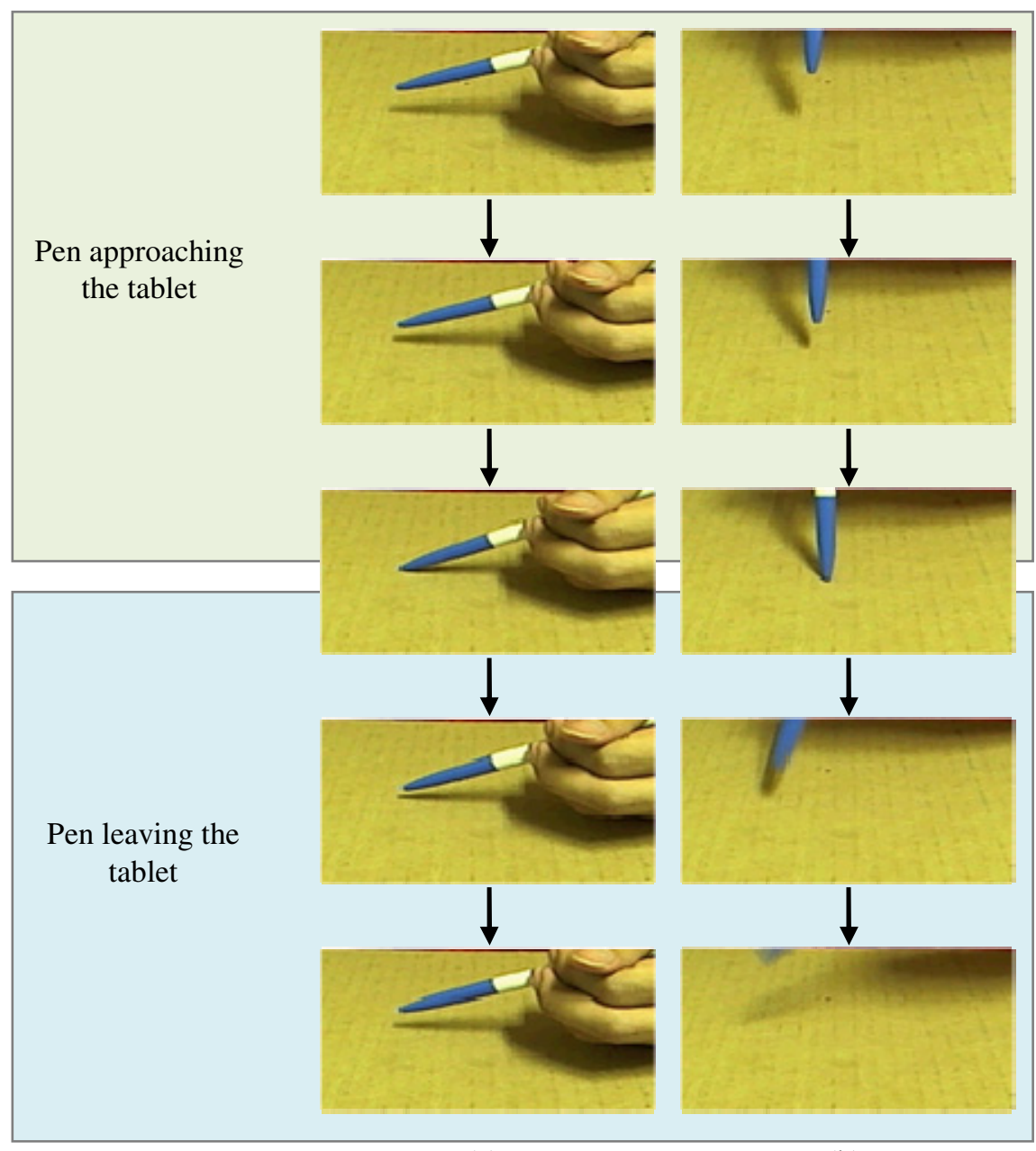

(a)

(b)

Fig. 3. A pen approaching a tablet and then leaving it: (a) the pen is tilted by 63 degree; (b) the pen is upright; that is, tilted by 0 degree 
As seen from Fig. 3, when the pen approaches and leaves the tablet, there is an interval that the pen approaches and leaves its shadow. This interval can be obtained from capturing a sequence of consecutive frames by calculating the distance between the pen and its shadow in each of the frames respectively. Equation (1) formulates the distance between the pen and its shadow, where $i$ stands for the index of a frame, $S B$ is the bounding box of the shadow, and $P B$ is the bounding box of the pen. The distance is acquired from Eq. (1), where $S B_{i}^{T o p}$ is the upper side of shadow's bounding box and $P B_{i}^{\text {Bottom }}$ is the bottom side of pen's bounding box as Fig. 4 shows.

$$
D(i)=S B_{i}^{T o p}-P B_{i}^{\text {Bottom }}
$$

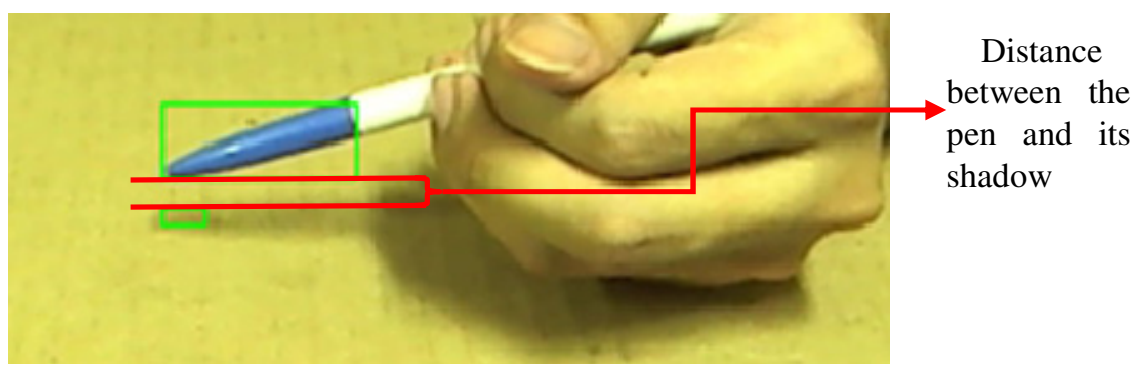

Fig. 4. Illustration of the distance between the pen and its shadow

Let $n$ be the index of the current frame, and $D(n)$ be the distance between the pen and its shadow in the current frame. We calculate the differences between $D(n)$ and $D(n-1), D(n-1)$ and $D(n-2), D(n-2)$ and $D(n-3)$, and so on. Then the average of the differences is computed, which is called the average distance variation $\bar{D}_{v a r}$, as expressed in Eq. (2), where $m$ is the number of frames needed for computation.

$$
\bar{D}_{\text {var }}=\frac{1}{m} \sum_{k=0}^{m-1} D(n-k)-D(n-k-1), \quad m \geq 2
$$

By means of the average distance variation, we can detect the pen action conducted by the user. This detection approach is depicted in Eq. (3) and stated as follows. Theoretically, $\bar{D}_{v a r}$ is positive when the pen is leaving the tablet. Conversely, $\bar{D}_{v a r}$ is negative when the pen is approaching the tablet. And $T_{a d v}$ is the threshold for detecting the variation, which is employed to discard frames with minor variation. If the variation is smaller than the threshold, the current result will not be updated.

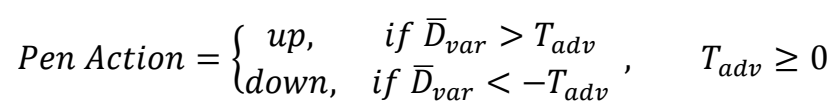

While the shadow detection is set to a very small area, we can deem these two actions as a pen touching and detaching the tablet, respectively. This is because when 
the detection area is very small, the pen needs to be put very close to the tablet. While the pen is quite near the shadow, it implies that the pen is very close to the tablet. Consequently, the characteristic of this behavior can be used for detecting a pen touching or detaching the tablet.

\section{Experimental Results and Discussions}

Many experiments of tablet detection, stylus detection, and pen shadow detection have been carried out to demonstrate the effectiveness of our proposed methods. Table 1 lists the developing environment for creating a virtual handwriting tablet system. In this experimental system, we adopt a webcam mounted on the monitor of a notebook computer, which is modeled Logitech's HD Pro Webcam C910 to support Full HD 1080p recording as shown in Fig. 5(a). The video camera is set to capture an image every $50 \mathrm{~ms}$, which means the FPS set to 20 . And the image resolution is set to $640 \times 480$.

Additionally, we take a traditional ball pen for emulating a digital stylus, and choose a piece of corrugated paper for simulating a tablet. The experimental set is shown in Fig. 5(b), where a piece of corrugated paper is put in front of the notebook computer, and a keyboard is placed between the piece of corrugated paper and the monitor. Figure 6(a) shows a real FOV of the camera, and the experimental ambient environment is illuminated by LED lights as shown in Fig. 6(b).

Table 1. The Developing Environment for Creating Our Virtual Handwriting Tablet System

\begin{tabular}{cc}
\hline & Hardware \\
\hline CPU & Intel(R) Core(TM) i7 CPU Q740 @ 1.73GHz 1.73GHz \\
\hline RAM & DDR3 4.00GB \\
\hline Operating System & Software \\
\hline & Microsoft Windows 7 Ultimate 32-bit \\
Developing Tools & Microsoft Visual Studio 2010 \\
& Microsoft .NET Framework 4 \\
C\# Language \\
OpenCV (EmguCV) 2.4.2
\end{tabular}

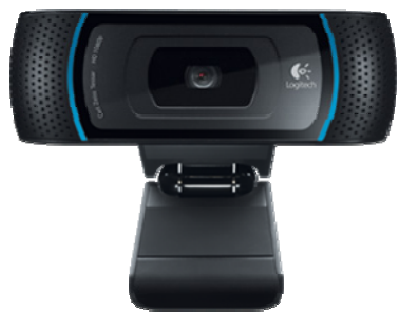

(a)

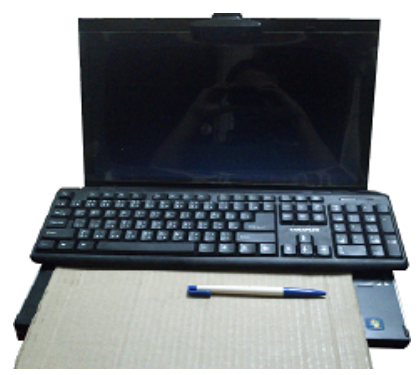

(b)

Fig. 5. Experimental equipment: (a) the used camera modeled Logitech HD Pro Webcam C910; (b) the set of our virtual handwriting tablet system 


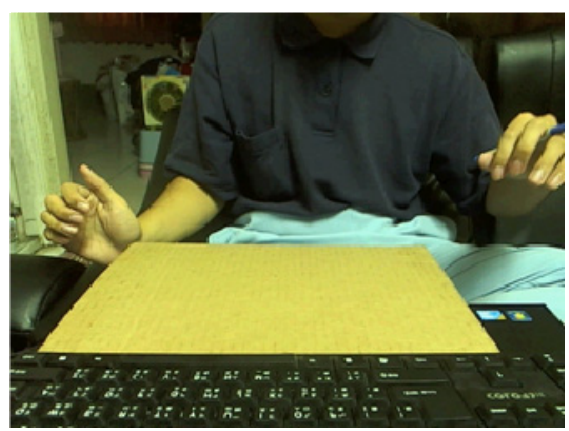

(a)

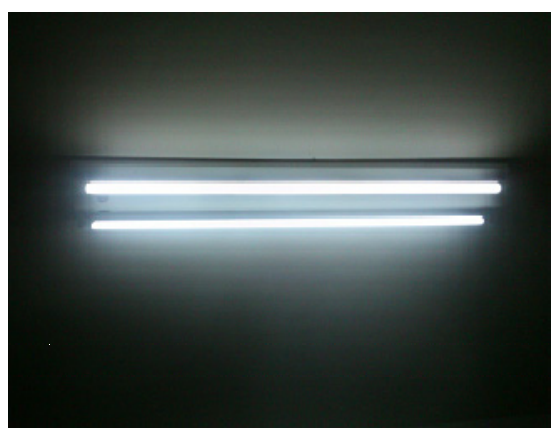

(b)

Fig. 6. Experimental environments: (a) an FOV seen from the webcam; (b) an ambiance illuminated by LED lights

Because our virtual handwriting tablet system takes advantage of pen's shadow for stylus detection, the position of a light source and the placement of its constituting components are very important. Figure 7 illustrates the deployment of the light source and each component of the system.

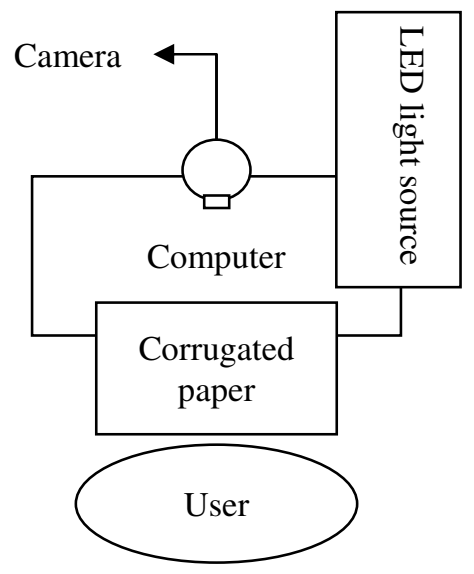

Fig. 7. The deployment of all the light source and components constituting the virtual handwriting tablet system

The following performs experiments on the methods proposed in Section 2 to detect pen moving directions with unlike parameters. The form of pen's shadow is varied along with different pen tilt angles as shown in Fig. 8. Therefore, we divide the pen tilt angles $\left(-80^{\circ}\right.$ to $\left.80^{\circ}\right)$ into five equal portions, each of which possesses an angle of 32 degrees; that is, ranged from $-80^{\circ}$ to $-48^{\circ}$, from $-48^{\circ}$ to $-16^{\circ}$, from $-16^{\circ}$ to $16^{\circ}$, from $16^{\circ}$ to $48^{\circ}$, and from $48^{\circ}$ to $80^{\circ}$. Then we make the pen approach and leave the tablet for 100 times under different pen tilt angles, where the distance between the pen and the tablet is about $1 \mathrm{~cm}$ when approaching and leaving. We record the detection rate for each of the above five ranges, respectively. The detection of pen moving directions is correct only when the shadow is detected accurately on both approaching and leaving at that time. We want to find which parameter achieves the best detection rate. 

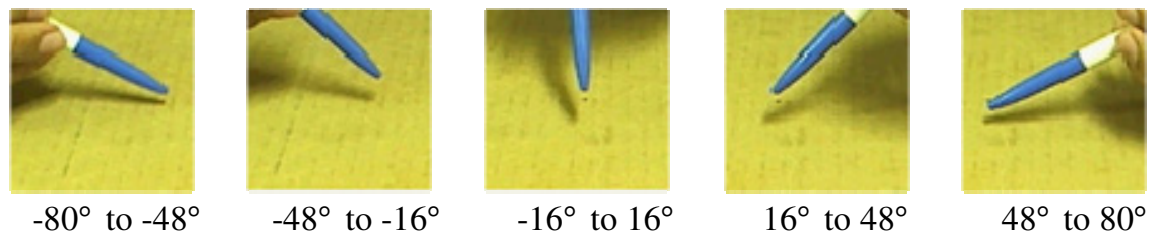

Fig. 8. Pen shadows varied with different pen tilt angles

Figure 9 shows the best detection result is acquired from the pen tilt angles between $-16^{\circ}$ and $16^{\circ}$. This is because the shadow appears in the side of the pen shown in Fig. 10 for these angles when the pen approaching and leaves the tablet.

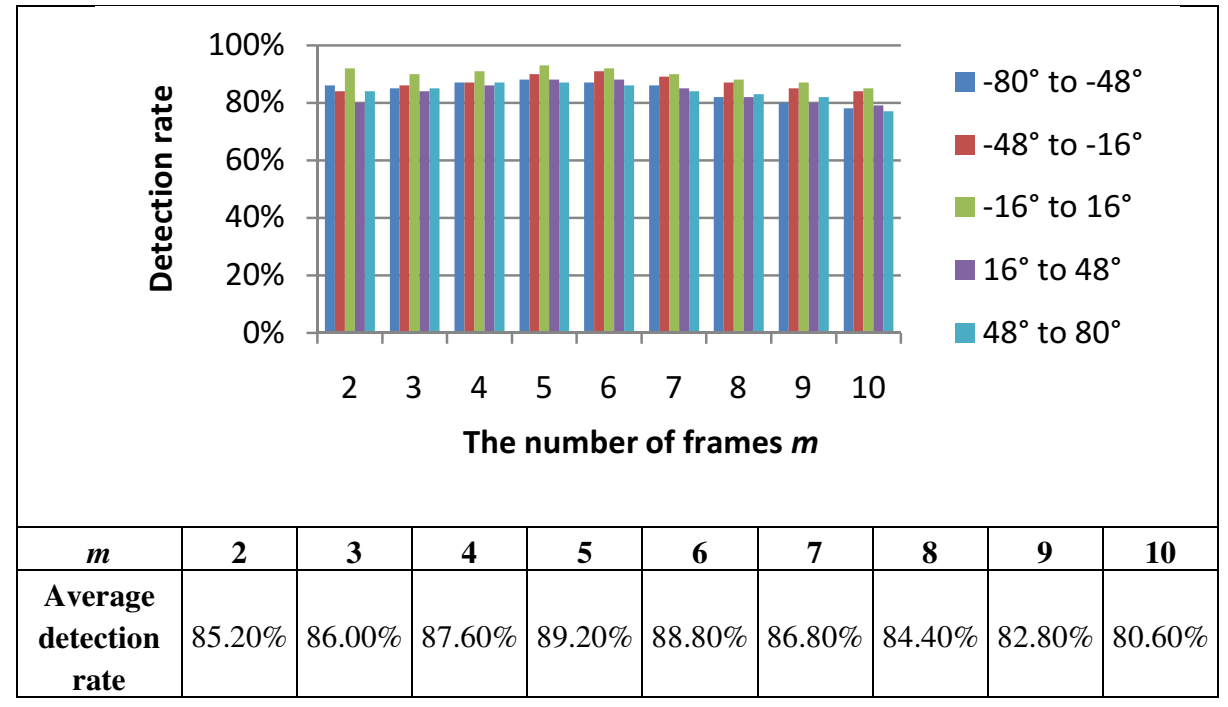

Fig. 9. The detection rate under different pen tilt angles for $m=2,3, \ldots, 10$

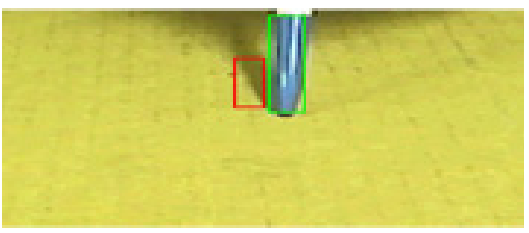

(a)

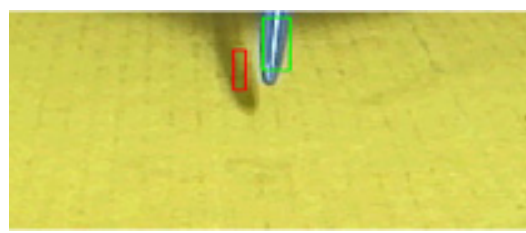

(b)

Fig. 10. The correct detection result: (a) the pen touching the tablet; (b) the pen leaving the tablet under its tilt angles between $-16^{\circ}$ and $16^{\circ}$ for $m=5$ 
Besides, it is easily found that the detection rate is higher when the degree of the pen tilt angle is negative. This is caused by the light source located at the upper right corner. As a result, the produced shadow in Fig. 11(a) is more saturated than that in Fig. 11(b), since in Fig. 11(a), the direction of the pen is perpendicular to that of the light source; conversely, in Fig. 11(b), the direction of the pen is parallel to that of the light source.

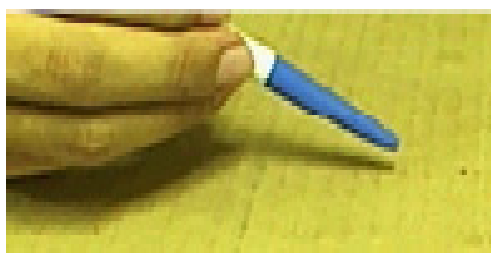

(a)

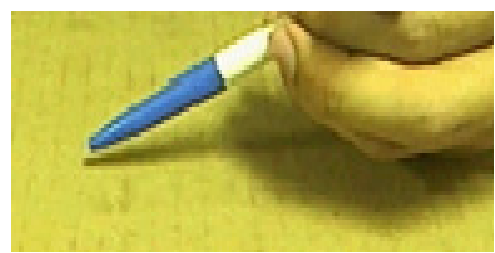

(b)

Fig. 11. Varied saturation of the produced shadow: (a) a more saturated shadow (the pen tilt angle $=-63.43^{\circ}$ ); (b) a less saturated shadow (the pen tilt angle $=63.43^{\circ}$ )

After observing the result, it is clear that the detection rate starts to decrease from $m=6$. We find this is owing to the relation between the FPS and user's pen movement speed. When the user moves the pen to leave the tablet needs 5 frames, but we set to 10 frames, the additional 5 frames decreases the average distance variation. On the contrary, if it is set to 2 frames, the pen will be detected to touch the tablet before it really does. In this case, any small movements of the pen will cause wrong detection. For the same FPS, if $m$ is small, the pen movement must be fast to reach an ideal detection rate. The best condition is that the number of frames for movement is equal to $m$.

\section{Conclusions and Future Works}

Nowadays, the human-computer interface is a popular research field, such as gesture recognition and virtual keyboard. These new interfaces bring users different kinds of experiences. Our proposed system is a new human-computer interface which adopts computer vision technology to imitate the function of a handwriting tablet, and improves the shortcomings that a traditional handwriting tablet may have; for example, not easy to carry, heavy weight, as well as fragile.

We have further proposed a method, which can detect a pen overlapping a tablet in the FOV of a camera. By detecting the variation of pen's shadows, the moving direction of a pen can be detected, which makes it possible to detect the pen touching a tablet with a single camera.

The system can be divided into three parts, including tablet selection, stylus detection, and pen shadow detection. The users select an object to be the tablet, and the system decides whether the selected object meets the conditions of a tablet. The system then finds the pen within the region of the tablet, and detects the position of the 
shadow of the pen. At last, the obtained information is saved for our system to send to the computer.

In the experiments, we employ different parameters for the proposed system, and analyze the experimental results. We find the optimal parameters and discuss about them. This paper has presented a novel human-computer interface and its applications. There are many research directions for the future work:

(1) Multi-touching

The proposed system can only detect one pen at a time. In the future, the system can be extended into multiple pen detection, in order to reach the goal of multi-touching.

(2) Right button click detection

Currently, our system is designed to emulate the movement of a mouse and the click of a left button. A right button click detection mechanism can be designed in the future.

(3) Detection by shadow only

Because the direction of a light source may vary, we cannot detect whether a pen has touched the tablet by pen shadow detection only. Therefore, we choose to set the range of capturing shadows to help detect the movement of the pen. However, this approach provides only a detection of the pen approximately touching a tablet. In consequence, we will intend to develop other effective methods to detect a pen touching a tablet.

(4) Writing assistance

Currently, we merely provide an object detection method. In the future, we will further combine a writing assistance system, which can help smooth the moving path, tablet calibration, as well as stop word detection.

Acknowledgement. The authors thank the National Science Council of Taiwan (R. O. C.) for supporting this work in part under Grant NSC-101-2221-E-011-143-MY3.

\section{References}

1. Wacom Intuos Pen I Wacom I Wacom Taiwan,

https: / / www. wacom.asia/intuos5/wacom-grip-pen

(accessed on January 12, 2014)

2. Intuos Pro I Wacom I Wacom Taiwan, https: / /www. wacom.asia/tw/intuos-pro (accessed on January 12, 2014)

3. Product List I Wacom I Wacom Taiwan, https://www.wacom.asia/tw/products-price (accessed on January 12, 2014) 